\title{
Understanding Convective Extreme Precipitation Scaling Using Observations and an Entraining Plume Model
}

\author{
JESSICA M. LORIAUX \\ Delft University of Technology, Delft, and Royal Netherlands Meteorological Institute, De Bilt, Netherlands \\ GEERT LENDERINK \\ Royal Netherlands Meteorological Institute, De Bilt, Netherlands \\ STEPHAN R. De RoOde \\ Delft University of Technology, Delft, Netherlands
}

\section{A. PIER Siebesma}

Delft University of Technology, Delft, and Royal Netherlands Meteorological Institute, De Bilt, Netherlands

(Manuscript received 26 November 2012, in final form 6 August 2013)

\begin{abstract}
Previously observed twice-Clausius-Clapeyron (2CC) scaling for extreme precipitation at hourly time scales has led to discussions about its origin. The robustness of this scaling is assessed by analyzing a subhourly dataset of 10-min resolution over the Netherlands. The results confirm the validity of the previously found 2CC scaling for extreme convective precipitation.

Using a simple entraining plume model, an idealized deep convective environmental temperature profile is perturbed to analyze extreme precipitation scaling from a frequently used relation based on the column condensation rate. The plume model simulates a steady precipitation increase that is greater than ClausiusClapeyron scaling (super-CC scaling). Precipitation intensity increase is shown to be controlled by a flux of moisture through the cloud base and in-cloud lateral moisture convergence. Decomposition of this scaling relation into a dominant thermodynamic and additional dynamic component allows for better understanding of the scaling and demonstrates the importance of vertical velocity in both dynamic and thermodynamic scaling. Furthermore, systematically increasing the environmental stability by adjusting the temperature perturbations from constant to moist adiabatic increase reveals a dependence of the scaling on the change in environmental stability. As the perturbations become increasingly close to moist adiabatic, the scaling found by the entraining plume model decreases to $\mathrm{CC}$ scaling. Thus, atmospheric stability changes, which are expected to be dependent on the latitude, may well play a key role in the behavior of precipitation extremes in the future climate.
\end{abstract}

\section{Introduction}

Events of convective extreme precipitation are highly disruptive to society and are likely to intensify with global warming (Pall et al. 2011; Min et al. 2011). With increasing temperatures, the moisture-holding capacity of the atmosphere increases. Therefore, when sufficient moisture

Corresponding author address: Jessica M. Loriaux, Delft University of Technology, P.O. Box 5048, 2628 CN, Delft, Netherlands.

E-mail: j.m.loriaux@tudelft.nl is available, precipitation extremes are expected to be thermodynamically related to surface temperature through the Clausius-Clapeyron relation of an approximately $6 \%-7 \%{ }^{\circ} \mathrm{C}^{-1}$ increase (Allen and Ingram 2002; Pall et al. 2007). We will refer to this rate of precipitation increase as CC scaling. However, dynamical processes may influence precipitation intensity leading to deviations from CC scaling (O'Gorman and Schneider 2009a; Emori and Brown 2005; Trenberth et al. 2003). With this research we try to assess the behavior of extreme convective precipitation intensity over the Netherlands and determine which processes are responsible for it. 
Observational studies of extreme precipitation scaling for different climatic regions have led to diverging results ranging from decreasing precipitation intensity with temperature (Maeda et al. 2012) to an intensity increase of up to 2 times CC (2CC) over the Netherlands (Lenderink and van Meijgaard 2008, 2010). Scaling exceeding the $\mathrm{CC}$ relation (super-CC scaling) followed by leveling off at $15^{\circ} \mathrm{C}$ is observed for convective precipitation by Berg and Haerter (2011). Other studies (Hardwick-Jones et al. 2010; Utsumi et al. 2011) show CC increase for temperatures up to approximately $25^{\circ} \mathrm{C}$, after which scaling becomes negative.

There may be several causes for these divergent scaling results, such as different dynamic behavior in different regions, but at least some of the varying results can be attributed to the moisture availability in the area. In moisture-deprived areas, the atmospheric moisture content does not necessarily increase with temperature, and the precipitation rate can decrease. Research has shown that using dewpoint temperature $T_{d}$ rather than temperature leads to more robust scaling results, with more uniform scaling over a larger range of $T_{d}$ (Lenderink et al. 2011; Lenderink and van Meijgaard 2010). These dewpoint temperature studies for the Netherlands (Lenderink et al. 2011; Lenderink and van Meijgaard 2010) and Hong Kong (Lenderink et al. 2011) consistently show CC scaling in the low $T_{d}$ range, with $2 \mathrm{CC}$ scaling for dewpoint temperatures up to approximately $22^{\circ} \mathrm{C}$ (Lenderink et al. 2011). For dewpoint temperatures beyond this, precipitation scaling displays a leveling-off behavior.

The 2CC scaling found by Lenderink and van Meijgaard (2008) occurred for temperatures above approximately $12^{\circ} \mathrm{C}$, using hourly precipitation extremes at De Bilt, Netherlands. At lower temperatures CC scaling applied. In correspondence to this article, Haerter and Berg (2009) have argued that the observed 2CC scaling might be a statistical artifact, caused by a shift from stratiform to convective precipitation extremes. Though similar scaling results were found using dewpoint temperature (Lenderink and van Meijgaard 2010; Lenderink et al. 2011), the validity of $2 \mathrm{CC}$ scaling for convective precipitation has not yet been resolved satisfactorily.

In this paper, we assess the validity of 2CC scaling over the Netherlands using daily, hourly, and 10-min observations, with the aim of resolving whether $2 \mathrm{CC}$ scaling applies for convective precipitation. Following Lenderink et al. (2011), this analysis is based on dewpoint temperature rather than temperature, for more robust results. Though the observational study is based on data from the current climate, the underlying assumption when using this observed scaling in a global warming scenario is that the relevant parameters behave the same in a future climate as in the current climate. Lenderink et al. (2011) have found good agreement between observed scaling relations and climate predictions. Therefore, in this study we will assume precipitation scaling found from observations to be applicable to a global warming scenario.

Apart from observational analyses, convective precipitation scaling has also been analyzed using general circulation models (GCMs), and nonhydrostatic highresolution models in which convection is resolved, rather than parameterized. Based on a set of GCMs, O'Gorman (2012) finds precipitation increases of approximately $10 \%{ }^{\circ} \mathrm{C}^{-1}$ with a $90 \%$ confidence interval of $6 \%-14 \%{ }^{\circ} \mathrm{C}^{-1}$ for tropical extremes. Cloud-resolving models (CRMs) with different resolutions show lower tropical extreme precipitation increases, consistent with CC scaling (Muller et al. 2011; Romps 2011).

Using an entraining plume model, we try to reproduce observed precipitation-scaling behavior by analyzing a condensation rate scaling relation similar to previously used scaling relations (e.g., O'Gorman and Schneider 2009a,b; Romps 2011; Muller et al. 2011). To understand the precipitation scaling better, we take a closer look at the processes behind it in the plume model, as well as the thermodynamic and dynamic contributions, and in particular the importance of the vertical velocity. Furthermore, we expect that some of the differences in scaling results might be attributed to latitudinal dependencies. While the thermal stratification in the tropics is expected to increase approximately moist adiabatically (e.g., Romps 2011), this is not clear for midlatitudes (Frierson 2006; Schneider and O'Gorman 2008). The influence of environmental changes on the increase of extreme intensities is investigated by perturbing the temperature profile based on a moist adiabatic to constant temperature increase.

One of the limitations of the plume model with respect to cloud-resolving models is that environmental conditions are fixed, and we are unaware of processes that might affect these conditions, such as convective organization. We recognize the limitations of the plume model, and emphasize that it is used merely as a conceptual tool, for better qualitative understanding of the processes determining precipitation scaling.

Summarizing, this article considers two main research questions:

(i) Is 2CC scaling robust for convective extreme precipitation over the Netherlands, or can previously found 2CC scaling results be ascribed to a statistical artifact?

(ii) Can we increase our conceptual understanding of extreme precipitation scaling using an entraining plume model?

The first question is dealt with in section 2 , in which the observational setup and results are presented. This is followed by the model description and experimental setup in 
section 3. The second question is treated in sections 4 and 5. In section 4 , a precipitation rate scaling relation is introduced, and plume model results are shown. Section 5 provides an analysis of thermal stratification changes. Finally, we present an overview of our findings in section 6 .

\section{Observations}

Previous research on precipitation extremes in the Netherlands has shown 2CC scaling behavior at an hourly resolution for (dewpoint) temperatures above a certain value (Lenderink and van Meijgaard 2008, 2010; Lenderink et al. 2011). At lower temperatures, CC scaling was found. Whether this unexpectedly high 2CC scaling is an intrinsic property of extreme convective precipitation or transitional behavior between two scaling regimes has been a point of discussion. Both points of view are related to the fact that precipitation events can be of stratiform or convective origin, and that both types have different characteristics. First, stratiform events have longer durations and lower intensities than convective events (Berg et al. 2013). Second, convective events generally take place at higher temperatures than stratiform events.

Lenderink and van Meijgaard believe that the 2CC scaling observed at higher temperatures for the hourly analysis is robust for convective extremes, while CC scaling, as found for the daily and lower-temperature hourly analyses, indicates extremes of a stratiform origin. They argue that, as a result of the typical time scales of stratiform and convective precipitation, at a daily resolution stratiform events dominate the extremes even at high temperatures, while at an hourly resolution, both convective and stratiform events are important. Since stratiform extremes are expected to dominate at lower temperatures than convective extremes, the bifurcation in hourly data between CC and 2CC scaling is interpreted as a stratiform-dominated CC scaling range and a convection-dominated $2 \mathrm{CC}$ scaling range.

However, based on the same characteristics, Haerter and Berg (2009) have argued that the observed 2CC scaling might be a statistical artifact, induced by the difference in intensities between stratiform and convective extremes. They suggest that stratiform and convective extreme events are both described by CC scaling, with consistently higher intensities for convective events. On an hourly time-scale, precipitation extremes are predominantly stratiform at low temperatures, while at high temperatures convective extremes become dominant. In the transition zone between these two precipitation types, enhanced CC scaling (super-CC) is expected. According to this explanation, the 2CC trend observed by Lenderink and van Meijgaard originates from a transition between stratiform-dominated and convection-dominated events.
Both hypotheses concur that the bifurcated scaling found by Lenderink and van Meijgaard for hourly precipitation extremes is somehow caused by a shift from stratiform to convective dominated extremes but differ on the interpretation of the 2CC scaling. The hypotheses are based on hourly and daily precipitation analyses. Following the same arguments, convective extremes are expected to dominate even at low dewpoint temperatures when subhourly data are used. If a transition zone is present in the subhourly data, it is thus expected at low dewpoint temperatures. Therefore, by analyzing precipitation extremes at subhourly resolution, we can clarify whether 2CC scaling is a property of convective extremes or if it is simply a transitional effect between two CC scaling regimes.

\section{a. Methods}

To examine how scaling of precipitation extremes changes for different time scales, the Royal Netherlands Meteorological Institute (KNMI) in situ precipitation data from 27 stations across the Netherlands are analyzed for three different temporal resolutions. The dataset with the highest temporal resolution is a 10-min dataset of precipitation data, with a length of 8 years. Because of the amount of stations, this set contains 216 years of data. For consistency hourly and daily datasets are also analyzed. The hourly dataset contains 16 years of precipitation data per station. A set of daily precipitation extremes is obtained by upscaling an hourly dataset by binning into groups of $24 \mathrm{~h}$. To ensure a large enough data pool, 31 years of data per station are used for this.

For each dataset, only wet intervals are considered. This is determined by a precipitation threshold of $0.1 \mathrm{~mm}$. Since scaling relations are more clear when dewpoint temperature rather than temperature is used (Lenderink et al. 2011), we will evaluate precipitation intensities with respect to dewpoint temperature. When analyzing the relation between precipitation intensities and dewpoint temperature, the dewpoint temperature used is measured $4 \mathrm{~h}$ prior to the event, to exclude any $T_{d}$ effects induced by the event itself. Results based on daily $T_{d}, T_{d} 2 \mathrm{~h}$ prior to the event, or instantaneous $T_{d}$ are consistent with, but slightly more noisy than, the results based on this method (not shown). The daily mean dewpoint temperature is used for the analysis of daily precipitation intensities. The precipitation data are binned in overlapping $1^{\circ} \mathrm{C} T_{d}$ bins of $2^{\circ} \mathrm{C}$ width.

For each bin, extreme precipitation is analyzed using three different percentiles (90th, 99th, and 99.9th) in the upper range of the distribution. These percentiles are determined by pct $=100(n-1 / 2) / N$, where $N$ is the sample size of the bin, and $n$ is the index of the sorted distribution vector corresponding to percentile (pct). To 
ensure individual solutions, the distributions need to consist of a minimum of $n=N$ data points. To determine the 90th percentile, there must therefore be at least 5 data points, while for the 99.9 th percentile a minimum of 500 data points are required. Bins with less than twice this amount of data points are discarded.

\section{b. Results}

Figure 1 shows the 90th, 99th, and 99.9th percentile of the precipitation intensity distributions with dewpoint temperature along the $x$ axis for daily, hourly, and 10-min resolution. Note the logarithmic $y$ axis, where precipitation intensity is given in millimeters per day, millimeters per hour, and millimeters per 10-min intervals for the daily, hourly, and 10-min resolutions, respectively. The dashed lines indicate an exponential $7 \%{ }^{\circ} \mathrm{C}^{-1}$ intensity increase, consistent with $\mathrm{CC}$ scaling for dewpoint temperatures close to $12^{\circ} \mathrm{C}$. The dotted lines indicate an exponential $14 \%{ }^{\circ} \mathrm{C}^{-1}$ increase of intensity, consistent with $2 \mathrm{CC}$ scaling in the same $T_{d}$ range. The gray shading shows the $95 \%$ confidence level determined by means of a Monte Carlo ensemble, by randomly dropping $10 \%$ of the data for each bin for 100 runs.

For all temporal resolutions, the 90th percentile is noisier and shows less clear scaling behavior than the higher percentiles. This is especially true for the subhourly resolution. It indicates that the scaling relations discussed here are valid only for the most extreme cases, while for less extreme events precipitation is no longer determined by moisture availability alone, leading to deviations from (2)CC scaling. The classification of convective versus stratiform events in this paper is based on the predominance of one type over the other for the highest intensities given a temporal resolution. As we move toward lower percentiles, the scaling is no longer determined by either stratiform or convective events.

Figure 1a shows the daily extremes. At this temporal resolution, stratiform extremes are expected to dominate over convective extremes. The plotted percentiles seem to follow CC scaling for all $T_{d}$. At an hourly resolution (Fig. 1b) we find a transition from CC scaling at $T_{d}<8^{\circ} \mathrm{C}$ to $2 \mathrm{CC}$ scaling at higher $T_{d}$. These findings are consistent with Lenderink and van Meijgaard (2008, 2010). To determine whether the $2 \mathrm{CC}$ scaling relation found for hourly extremes is robust or a statistical artifact as proposed by Haerter and Berg, we turn to 10-min precipitation (Fig. 1c). At this temporal resolution, convective extremes dominate stratiform extremes even at low temperatures. Therefore, if 2CC scaling is indeed caused by a shift from stratiform to convective extremes, the transition zone should be present at lower dewpoint temperatures than in the hourly analysis and show CC scaling over most of the domain. However, the 99.9th

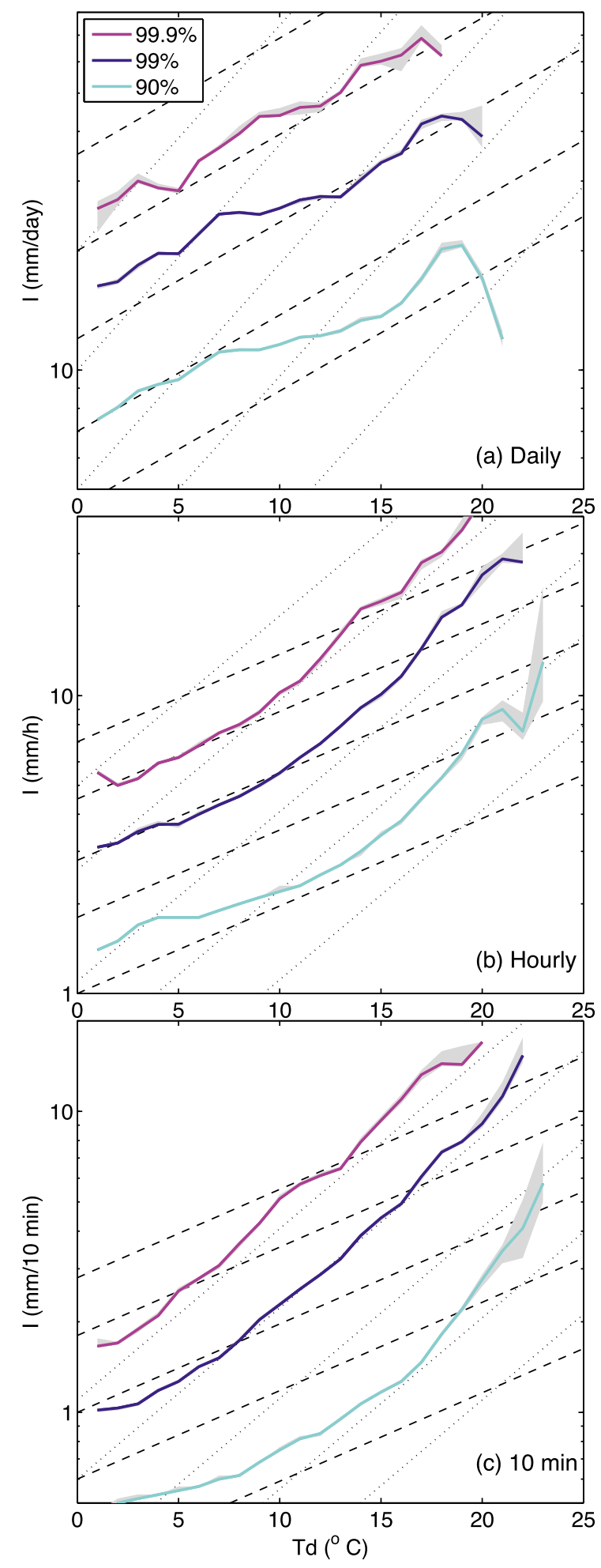

FIG. 1. Observed precipitation intensity for the 90th, 99th, and 99.9th percentiles, with respect to dewpoint temperature at (a) daily, (b) hourly, and (c) 10-min resolution. Gray shading indicates the $95 \%$ confidence interval. Dashed lines indicate a CC intensity increase, while dotted lines indicate a 2CC increase. Analysis is performed following Lenderink et al. (2011). 
percentile displays 2CC scaling over the full range of dewpoint temperatures, as does the 99th percentile for dewpoint temperatures above approximately $7^{\circ} \mathrm{C}$. This demonstrates that the observed shift in scaling is not caused by a transition between two regimes with CC scaling. Instead, while CC scaling seems applicable for stratiform precipitation, 2CC scaling appears to be an intrinsic property of convective precipitation extremes.

\section{The entraining plume model}

The convective scaling found from observations will be further analyzed using an entraining plume model based on moist static energy and total water content. Because of its simplicity, the model is very useful as a conceptual tool, and therefore lends itself to the purpose of gaining insight in the processes behind precipitation scaling.

The bulk plume model that we use is standard and is identical to many steady-state models that form the basis of many moist convection parameterizations in stratiform models (Tiedtke 1989; Siebesma and Holtslag 1996). In terms of the dry static energy $s=c_{p} T+g z$, specific humidity $q_{v}$ and liquid water $q_{l}$, it can be formulated as

$$
\begin{aligned}
w_{c} \frac{\partial s_{c}}{\partial z} & =L_{v} c-\epsilon w_{c}\left(s_{c}-s_{e}\right), \\
w_{c} \frac{\partial q_{v, c}}{\partial z} & =-c-\epsilon w_{c}\left(q_{v, c}-q_{v, e}\right), \quad \text { and } \\
w_{c} \frac{\partial q_{l, c}}{\partial z} & =c-G-\epsilon w_{c} q_{l, c},
\end{aligned}
$$

where $w_{c}$ is the updraft velocity, $c$ is the net condensation rate, and $G$ is the autoconversion from $q_{l}$ to precipitable water. In the equations above, subscript $c$ refers to the in-cloud values, while subscript $e$ indicates the environment. The entrainment rate $\epsilon$ can be set to zero for an undiluted plume. For a diluted plume, a value of $\epsilon=\max (0.4 / z)\left(10^{-4} \mathrm{~m}^{-1}\right)$ is used (e.g., Siebesma 1998; de Rooy and Siebesma 2008; Holloway and Neelin 2009). This set of equations can be recombined into equations for moist static energy $\left(s_{m, c}=s_{c}+L_{v} q_{v, c}\right)$ and total water specific humidity $\left(q_{t, c}=q_{v, c}+q_{l, c}\right)$ in the cloud. These parameters, in combination with pressure $p$, describe the thermodynamic state of the atmosphere. The equations for $s_{m, c}$ and $q_{t, c}$ become

$$
w_{c} \frac{\partial s_{m, c}}{\partial z}=-\epsilon w_{c}\left(s_{m, c}-s_{m, e}\right) \quad \text { and }
$$

$$
w_{c} \frac{\partial q_{t, c}}{\partial z}=-G-\epsilon w_{c}\left(q_{t, c}-q_{t, e}\right) .
$$

The equations above illustrate how at steady state the advection of $s_{m, c}$ and $q_{t, c}$ is balanced by autoconversion and lateral entrainment. Assuming zero evaporation of rain, the surface precipitation rate $P$ is related to the autoconversion rate through

$$
P=\int_{z_{b}}^{z_{t}} G \rho d z
$$

Here, $z_{b}$ is the cloud-base height: the lifting condensation level (LCL). The cloud top $z_{t}$ is the height at which the vertical velocity becomes zero. Since we are interested in extreme precipitation intensities, we assume that the maximum amount of precipitation that can fall out of an atmospheric column is determined by the total amount of condensation in the column (Iribarne and Godson 1981), so that $q_{l, c}=0$ and $c=G$. This assumption relates the precipitation intensity directly to the condensation rate [see (2)].

In the subcloud layer, lateral entrainment is assumed to be to zero. Hence, $s_{m, c}$ and $q_{t, c}$ are conserved in the subcloud layer below the LCL. Above the LCL, $q_{t, c}$ is bounded by $q_{s, c}$ as prescribed by the Clausius-Clapeyron equation. ${ }^{1}$ To solve these equations, the vertical velocity $w_{c}$ is computed from buoyancy $B$ and the entrainment, using

$$
\frac{1}{2} \frac{\partial w_{c}^{2}}{\partial z}=-\alpha_{1} \epsilon w_{c}^{2}+\alpha_{2} B, \text { where } B=g \frac{T_{v, c}-T_{v, e}}{T_{v, e}} .
$$

Here, $\alpha_{1}=1$ and $\alpha_{2}=0.6$ are constants (de Roode et al. 2012). The buoyancy is computed from the difference between cloud and environmental virtual temperature $T_{v}$ and gravitational constant $g$.

The model is implemented on a $20-\mathrm{m}$ vertical grid. Equations (4), (5), and (7) are solved in an upward loop using a first-order implicit discretization in the vertical. This process continues until the vertical velocity vanishes and the cloud top is reached.

The environmental profile used is based on a database of soundings taken twice a day between 1995 and September 2011 at De Bilt. From these soundings, 10 profiles with the highest undiluted, pseudoadiabatic convective available potential energy (CAPE) have been combined by taking the mean temperature and relative humidity (RH) at each level, resulting in a typical profile for deep

\footnotetext{
${ }^{1}$ The Clausius-Clapeyron equation can be written as $d e_{s} / e_{s}=L_{v} /$ $R_{v} d T / T^{2}$, where $e_{s}$ is the saturation vapor pressure, and $L_{v}$ and $R_{v}$ are the latent heat of vaporization and the gas constant, respectively.
} 


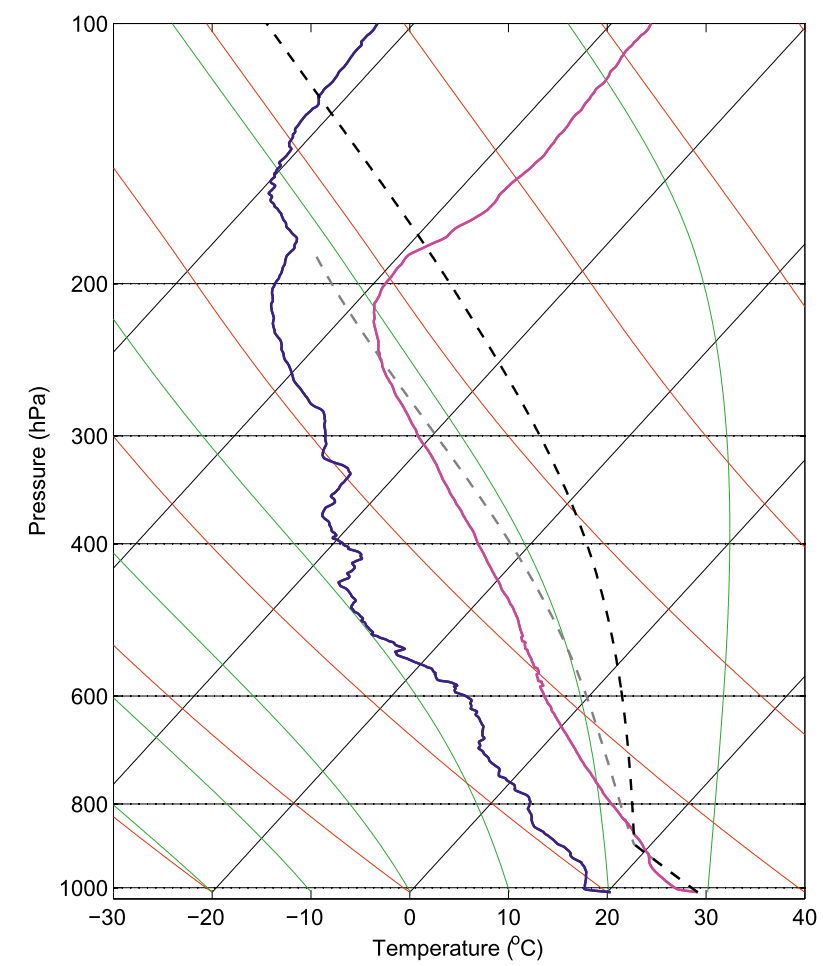

FIG. 2. Idealized environmental temperature (pink) and dewpoint temperature (blue) profiles. The updraft profiles for diluted and undiluted ascent are shown by the dashed gray and black lines, respectively. Skewed black lines are lines of equal temperature. The red lines indicate dry adiabats, while the green lines give the moist adiabats.

convective circumstances. The temperature and moisture conditions of this idealized profile, as well as the parcel ascent, are shown in Fig. 2. In this figure, the dark blue line indicates the dewpoint temperature profile, while the temperature is depicted in pink. Skewed black lines are lines of constant temperature, red lines represent dry adiabats, and green lines represent the moist adiabats. The undiluted ascent is depicted by the dashed black line and the diluted ascent is depicted by the dashed gray line. In Fig. 3, the diluted and undiluted profiles of $s_{m, c}$ and $q_{v, c}$ are plotted against the environmental variables. For undiluted ascent, $s_{m, c}$ is conserved, since it is not affected by condensation. The diluted updraft profile decreases upon reaching cloud base as a result of lateral entrainment. The figure shows that, for both diluted and undiluted ascent, the updraft profiles are unstable and deep. The $q_{v, c}$ profiles (right panel) are affected by the condensation rate. The influence of condensation is the difference between $q_{v, c}$ at cloud base and the undiluted $q_{v, c}$; the difference between the diluted and undiluted $q_{v, c}$ shows the influence of entrainment. It is clear that the condensation rate has a larger effect on the profiles than lateral entrainment does.
To analyze precipitation-scaling behavior, the environmental temperature profile is perturbed by $-3^{\circ}$ to $+3^{\circ} \mathrm{C}$ in steps of $1^{\circ} \mathrm{C}$. These perturbations occur under the assumption of constant relative humidity (Sherwood et al. 2010), so that at each level for a $1^{\circ} \mathrm{C}$ temperature increase, $T_{d}$ increases following $\mathrm{RH}=e_{s}(T) / e_{s}\left(T_{d}\right)$. Similar temperature perturbations are carried out in a recent precipitation study by Singleton and Toumi (2012). The sensitivity to stability changes is analyzed in section 5 .

Figure 4 shows diluted parcel profiles of buoyancy, vertical velocity, and condensation, for each perturbation. All three parameters increase at all levels for increasing temperature perturbations. The weight of the profiles moves upward. For example, the maximum condensation rate not only increases, but also shifts toward higher altitudes for increasing temperature.

Perturbed profiles of the saturation specific humidity and its gradient are shown in Fig. 5. The $q_{s}$ profile decreases exponentially with height. With increasing temperature perturbations, the moisture increases at all heights. The saturation specific humidity gradient $\partial q_{s} / \partial z$ also becomes stronger with increasing perturbations. Since there is hardly any moisture at higher levels, the moisture gradient is most pronounced at low levels, weakening with height.

Analysis of the changes in the perturbed profiles allows for the scaling of model output with temperature. The scaling of a variable $X$ is written as $\delta X / X$, the fractional rate of change. Here, the numerator gives the difference between the perturbed and reference value. The denominator gives the reference value. Rather than using the initial profile as a reference, we have chosen to use the lowest temperature perturbation $\left(-3^{\circ} \mathrm{C}\right)$ instead to ensure a continuous scaling range. This has not affected scaling results. Following Hardwick-Jones et al. (2010), the rate of change per degree is computed from

$$
\left(\frac{\delta X}{X}+1\right)^{1 / \Delta T}-1
$$

where we assumed an exponential relation. Here, $\Delta T$ is the temperature difference with respect to the reference value.

\section{Precipitation scaling}

In section 2 it was shown that $2 \mathrm{CC}$ scaling is robust for convective extremes over the Netherlands, which suggests that this behavior is governed by a few basic processes. The aim of the plume model experiment is to analyze the modeled precipitation-scaling behavior in order to assess the consistency with the observations 

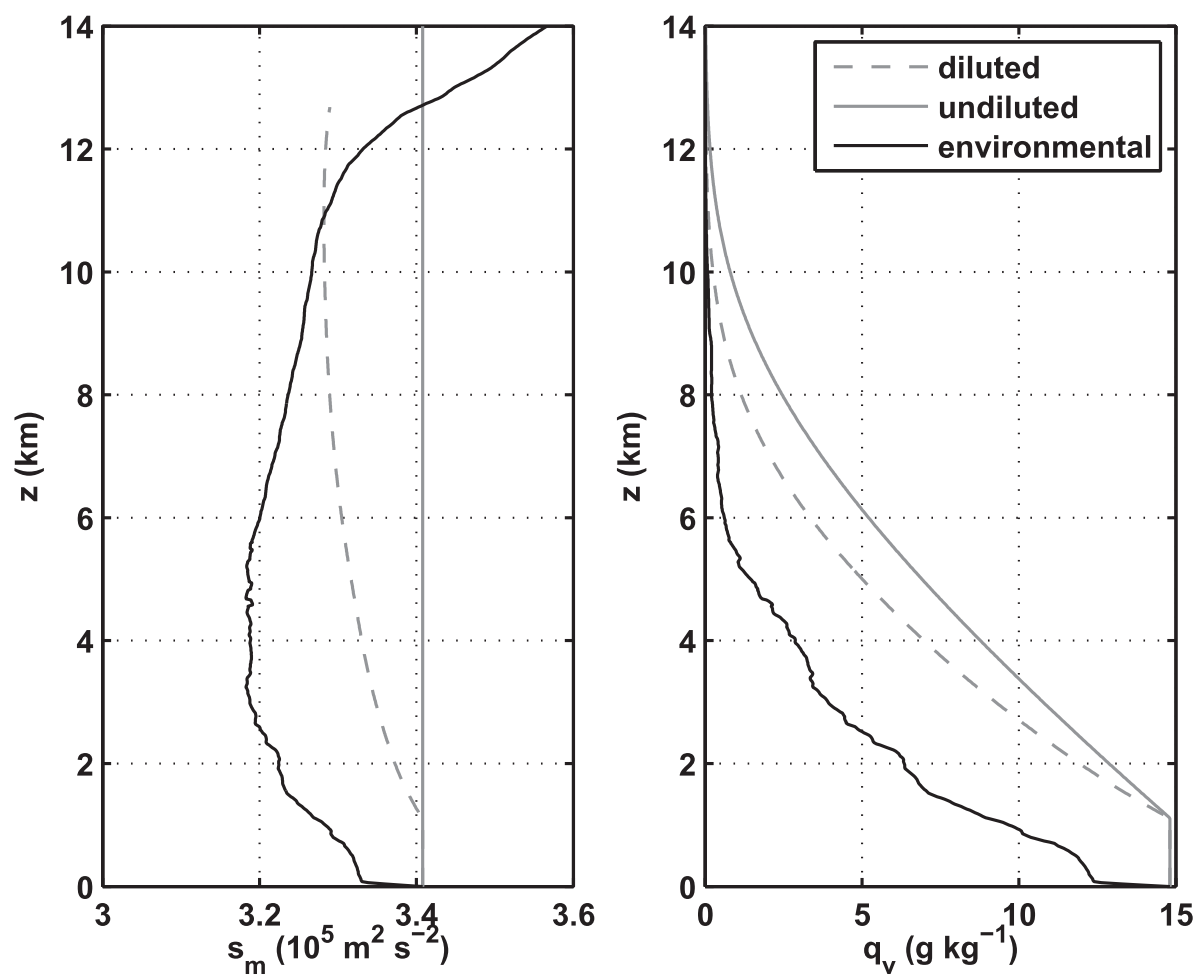

FIG. 3. Environmental and modeled profiles of (left) moist static energy and (right) specific humidity.

made in section 2 and to further develop our understanding of this behavior. Since the autoconversion is approximated by the rate of condensation, the columnintegrated precipitation rate $P$ follows directly from the condensation rate:

$$
\begin{aligned}
P & =[c], \\
& =-\left[w_{c}\left\{\frac{\partial q_{s, c}}{\partial z}+\epsilon\left(q_{t, c}-q_{t, e}\right)\right\}\right],
\end{aligned}
$$

where for simplicity we use $[\cdot]=\int_{z_{b}}^{z_{t}} \rho d z$. The precipitation rate is thus determined from the vertical advection of saturation specific humidity, corrected for entrainment.

To determine precipitation-scaling behavior due to temperature perturbations of the initial profile, we take the fractional rate of change of $P$

$$
\frac{\delta P}{P}=\frac{\delta\left[w_{c} \frac{\partial q_{s, c}}{\partial z}\right]+\delta\left[w_{c} \epsilon\left(q_{t, c}-q_{t, e}\right)\right]}{\left[w_{c}\left\{\frac{\partial q_{s, c}}{\partial z}+\epsilon\left(q_{t, c}-q_{t, e}\right)\right\}\right]} .
$$

The undiluted form of this equation $(\epsilon=0)$ has been frequently used for extreme precipitation analysis (e.g., Muller et al. 2011; O'Gorman and Schneider 2009a,b; Romps 2011). In Fig. 6, the precipitation scaling for diluted and undiluted plumes are shown in blue. Auxiliary lines for $\mathrm{CC}$ and 2CC scaling are also included. They are computed from surface $q_{s}$ using $T_{d}$, which approximates CC scaling at cloud base, since the parcel rises undiluted from the surface to this height. The fractional rate of change of $P$ shows super-CC scaling slightly below $10 \%{ }^{\circ} \mathrm{C}^{-1}$ and varies little with perturbations, indicating robust scaling behavior. Furthermore, the difference in scaling behavior between diluted and undiluted plumes is small. From this, we conclude that the fractional rate of change of $P$ is hardly affected by the environment. For simplicity, we therefore continue the scaling analysis for the undiluted case.

\section{a. Lateral versus cloud-base influence on precipitation increase}

By rewriting (9) for $\epsilon=0$, the two processes responsible for the scaling behavior of $P$ appear:

$$
P=\left.w_{c} \rho q_{s, c}\right|_{z_{b}}+\int_{z_{b}}^{z_{t}} \frac{\partial\left(\rho w_{c}\right)}{\partial z} q_{s, c} d z
$$

The first rhs term is the moisture flux at cloud base $P_{\mathrm{cb}}$. The moisture flux at cloud top has been neglected, since vertical velocity is zero at this height, and $q_{s}$ decreases exponentially with height. The second rhs term gives the column-integrated lateral cloud moisture convergence $P_{\mathrm{lm}}$. So, precipitation rate scaling is determined by the 

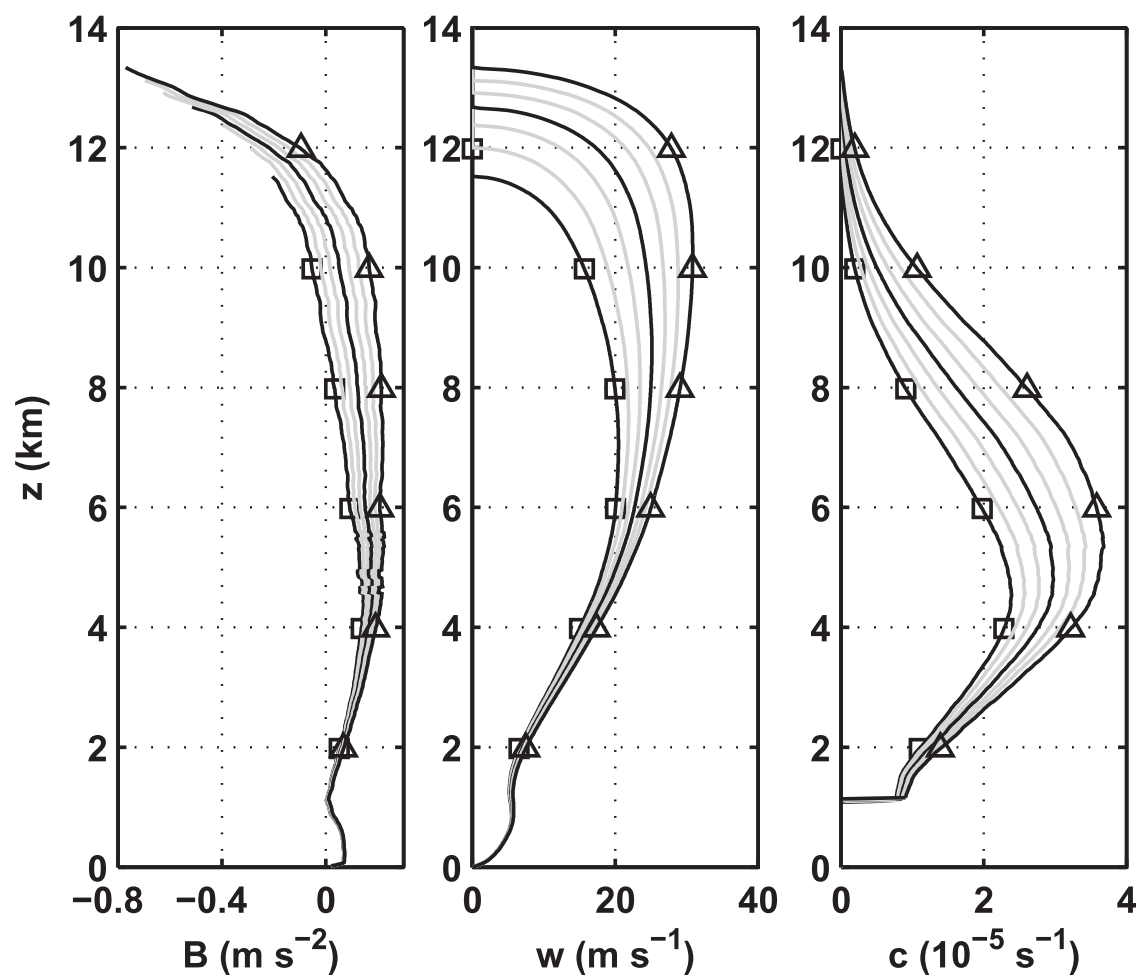

FIG. 4. Evolution of (left) diluted buoyancy, (middle) vertical velocity, and (right) condensation rate profiles with temperature perturbations from $-3^{\circ}$ (black squares) to $+3^{\circ} \mathrm{C}$ (black triangles) to the environmental profile. The reference profile is depicted by the black line in the middle, while gray lines depict the remaining perturbations between $-3^{\circ}$ and $+3^{\circ} \mathrm{C}$.

moisture flux at cloud base and the lateral moisture convergence. This is also depicted in Fig. 7. To understand how these processes contribute to the precipitation intensity scaling, we substitute (11) into the undiluted form of (10)

$$
\frac{\delta P}{P}=\frac{\delta P_{\mathrm{cb}}}{P}+\frac{\delta P_{\mathrm{lm}}}{P}
$$

Each rhs term gives the total contribution to the $10 \%{ }^{\circ} \mathrm{C}^{-1}$ increase found for precipitation rate scaling. For a better understanding of the behavior of both terms, we rewrite (12), so that

$$
\frac{\delta P}{P}=\frac{P_{\mathrm{cb}}}{P} \frac{\delta P_{\mathrm{cb}}}{P_{\mathrm{cb}}}+\frac{P_{\mathrm{lm}}}{P} \frac{\delta P_{\mathrm{lm}}}{P_{\mathrm{lm}}} .
$$

For each rhs term, the first part of the decomposition gives the relative importance of the process to the total precipitation scaling through the initial relative contribution of the process $\left(P_{\mathrm{cb}} / P\right.$ and $P_{\mathrm{lm}} / P$, respectively). The second part of the rhs terms gives the fractional rate of change of the process $\left(\delta P_{\mathrm{cb}} / P_{\mathrm{cb}}\right.$ and $\left.\delta P_{\mathrm{lm}} / P_{\mathrm{lm}}\right)$.

Figure 7 shows how these processes contribute to the rate of increase of precipitation intensity. With a relative contribution of $54 \%$, the lateral moisture convergence has a slightly larger influence on the precipitation scaling than the moisture flux at cloud base (46\%). Both processes are thus of almost equal importance to precipitationscaling behavior. Scaling of the cloud-base moisture flux is shown in Fig. 6 (light blue). With an approximate increase of $7 \%{ }^{\circ} \mathrm{C}^{-1}$, it exceeds $\mathrm{CC}$ scaling by close to $1 \%{ }^{\circ} \mathrm{C}^{-1}$. Scaling of the lateral moisture flux approaches an increase close to $2 \mathrm{CC}$ scaling (Fig. 6, pink). Following (13), we can indeed explain the precipitation scaling of $10 \%{ }^{\circ} \mathrm{C}^{-1}$ as found from the model by weighing the scaling of both the cloud base and lateral moisture fluxes with their initial magnitude, since

$$
\frac{\delta P}{P} \approx 0.46 \times 6.7+0.54 \times 12.5 \approx 10 \%{ }^{\circ} \mathrm{C}^{-1} \text {. }
$$

\section{b. Analysis of the role of (thermo-) dynamics and the vertical velocity}

Assuming changes in density with perturbations to be small, scaling of the cloud-base moisture flux can be understood in terms of thermodynamic and dynamic changes. The thermodynamic changes are computed by analyzing temperature and humidity variations using 

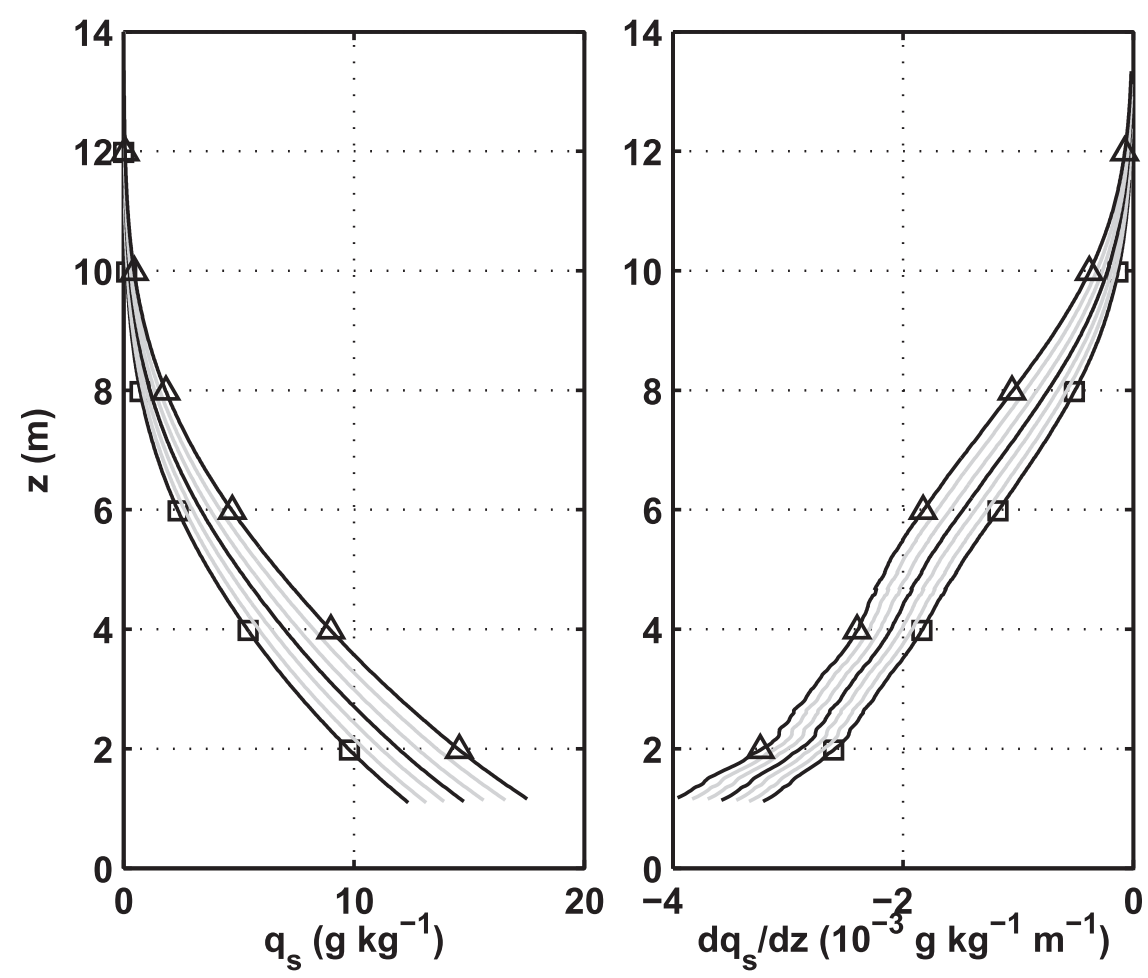

FIG. 5. Evolution of (left) diluted saturation specific humidity and (right) $\partial q_{s} / \partial z$ with temperature perturbations from $-3^{\circ}$ (black squares) to $+3^{\circ} \mathrm{C}$ (black triangles) to the environmental profile. The reference profile is depicted by the black line in the middle, while the gray lines depict the remaining perturbations between $-3^{\circ}$ and $+3^{\circ} \mathrm{C}$.

the velocity field from the reference profiles, whereas dynamic changes are computed by analyzing the changes in velocity, while using the temperature and humidity profiles from the reference profile:

$$
\delta P_{\mathrm{cb}}=\delta P_{\mathrm{cb}, \mathrm{th}}+\delta P_{\mathrm{cb}, d},
$$

where $P_{\mathrm{cb}, \mathrm{th}}=\left.w_{c} \rho \delta\left(q_{s, c}\right)\right|_{z_{b}}$ and $P_{\mathrm{cb}, d}=\left.\delta\left(w_{c}\right) \rho q_{s, c}\right|_{z_{b}}$ represent the thermodynamic and dynamic components of $P_{\mathrm{cb}}$, respectively. The thermodynamic scaling contribution equals $\mathrm{CC}$ scaling at cloud base, which can be approximated by $\mathrm{CC}$ scaling with surface $T_{d}$. The vertical velocity at cloud base also increases with increasing temperature perturbations owing to increased buoyancy. This accounts for another approximately $1 \%{ }^{\circ} \mathrm{C}^{-1}$ scaling, leading to the small exceedance of $\mathrm{CC}$ scaling. The results of this decomposition into thermodynamic and dynamic scaling can be found in Fig. 8.

Analogous to the cloud-base moisture flux, the integrated lateral moisture convergence scaling is also governed by thermodynamic and dynamic changes, so that

$$
\delta P_{\mathrm{lm}}=\delta P_{\mathrm{lm}, \mathrm{th}}+\delta P_{\mathrm{lm}, d},
$$

where $P_{\mathrm{lm}, \mathrm{th}}=\int_{z_{b}}^{z_{t}} \delta\left(q_{s, c}\right) \partial\left(\rho w_{c}\right) / \partial z d z$ is the thermodynamic and $P_{\mathrm{lm}, d}=\int_{z_{b}}^{z_{t}} q_{s, c} \partial\left\{\rho \delta\left(w_{c}\right)\right\} / \partial z d z$ is the dynamic component of $P_{\mathrm{lm}}$. With an increase close to $8 \%{ }^{\circ} \mathrm{C}^{-1}$, the thermodynamic contribution (first rhs term) exceeds $\mathrm{CC}$ scaling. Bearing in mind the column-integrated nature of the lateral moisture convergence, it is not surprising that the thermodynamic increase lies closer to the increase of column-integrated $q_{s}$, or water vapor path (WVP) scaling of $9 \%{ }^{\circ} \mathrm{C}^{-1}$, than CC scaling as determined from surface values. The dynamic contribution (second rhs term) is positive and accounts for another $5 \%{ }^{\circ} \mathrm{C}^{-1}$ of the lateral moisture convergence scaling (Fig. 8).

Now that we have an analysis of the thermodynamic and dynamic changes per process, we can also assess the total thermodynamic and dynamic contributions to the precipitation, where

$$
\frac{\delta P}{P}=\frac{\delta P_{\mathrm{th}}}{P}+\frac{\delta P_{d}}{P} .
$$

Here, $P_{\text {th }}$ and $P_{d}$ denote the thermodynamic and dynamic scaling contributions, respectively. Note that the relative contributions of the integrated lateral $(54 \%)$ and cloud-base $(46 \%)$ moisture flux are unchanged for 


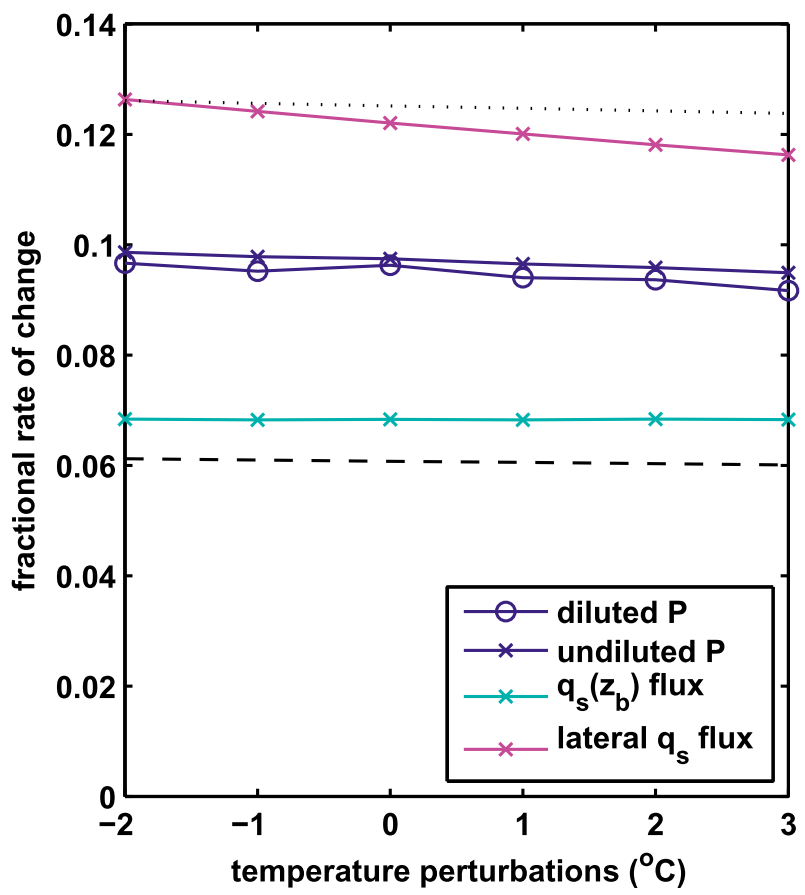

FIG. 6. The fractional rate of change $\left({ }^{\circ} \mathrm{C}^{-1}\right)$ of diluted and undiluted precipitation rate in blue. Scaling of the undiluted cloudbase moisture flux and the integrated lateral moisture convergence are shown in light blue and pink, respectively. For illustration purposes $\mathrm{CC}$ (dashed black) and 2CC (dotted black) scaling are also included.

thermodynamic and dynamic decomposition. Therefore, following (13), thermodynamic and dynamic contributions become

$$
\begin{gathered}
\frac{\delta P_{\mathrm{th}}}{P}=\frac{P_{\mathrm{cb}}}{P} \frac{\delta P_{\mathrm{cb}, \mathrm{th}}}{P_{\mathrm{cb}}}+\frac{P_{\mathrm{lm}}}{P} \frac{\delta P_{\mathrm{lm}, \mathrm{th}}}{P_{\mathrm{lm}}} \\
\approx 0.54 \times 8+0.46 \times 6=7 \%{ }^{\circ} \mathrm{C}^{-1}, \text { and } \\
\frac{\delta P_{d}}{P}=\frac{P_{\mathrm{cb}}}{P} \frac{\delta P_{\mathrm{cb}, d}}{P_{\mathrm{cb}}}+\frac{P_{\mathrm{lm}}}{P} \frac{\delta P_{\mathrm{lm}, d}}{P_{\mathrm{lm}}} \\
\approx 0.54 \times 5+0.46 \times 0.7=3 \%{ }^{\circ} \mathrm{C}^{-1},
\end{gathered}
$$

respectively (Fig. 8). This shows that changes in thermodynamics account for most of the scaling, with slight exceedance of surface CC scaling. This exceedance is caused by the column-integrated moisture convergence. The super-CC scaling found from the model is mostly caused by the dynamic contribution, which adds an additional $3 \%{ }^{\circ} \mathrm{C}^{-1}$ to the precipitation intensity scaling. This signifies the important role of vertical velocity changes in precipitation intensity scaling.

Note, however, that apart from the dynamic contribution the reference vertical velocity profile also determines

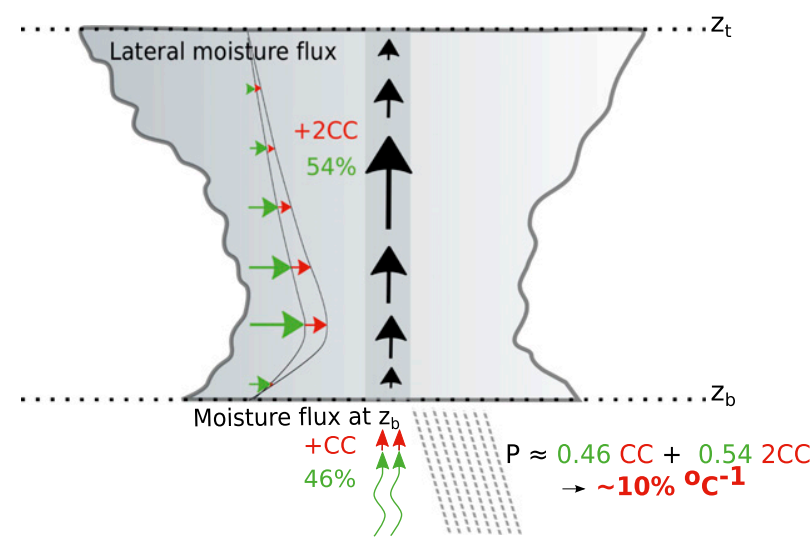

FIG. 7. Cartoon describing how precipitation intensity scaling as found from the plume model is built up from lateral and cloud-base moisture fluxes. Green is used to indicate the initial state, while scaling is depicted in red.

the relative magnitude of the column-integrated and cloud-base moisture fluxes. Depending on the shape and magnitude of the vertical velocity profile, the balance between the column-integrated moisture convergence and the cloud-base moisture flux is determined. Though thermodynamics make up for the largest part of precipitation scaling, this portrays the important role of the

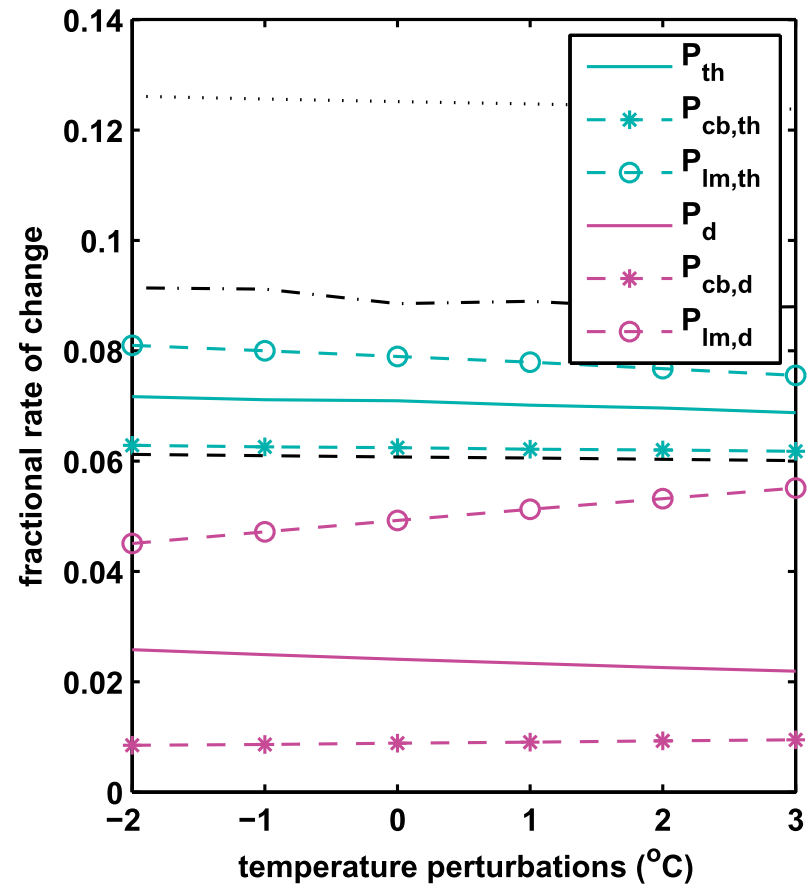

FIG. 8. The fractional rate of change $\left({ }^{\circ} \mathrm{C}^{-1}\right)$ of the thermodynamic (light blue) and dynamic (pink) scaling contributions, as well as the cloud-base and lateral (thermo-) dynamic components. For illustration purposes CC (dashed black), WVP (dash-dotted black), and 2CC (dotted black) scaling are also included. 
vertical velocity in precipitation intensity scaling, not only through the dynamic contribution, but also in determining which process has the largest influence on the scaling.

\section{c. Robustness of the results}

To ensure the robustness of the experiment results, the experiment was performed using several modifications to the deep convective profile regarding the environmental moisture. These profile variations yielded similar results to those discussed in this paper. The experiment was also repeated using the deep convective Weisman and Klemp (1982) profiles. With a precipitation increase of around $9 \%{ }^{\circ} \mathrm{C}^{-1}$, these results are consistent with the results presented in this article. Furthermore, rather than using the assumption that $G=c$, we also implemented several frequently used autoconversion schemes. Condensation scaling when using Kessler autoconversion (Kessler 1969), as well as more advanced formulations such as proposed by Sundqvist (1978) with and without ice microphysics, did not significantly alter the results. This puts the results found in this section in stronger footing.

\section{Scaling sensitivity to environmental stability}

In the tropics, the thermal stratification of the atmosphere is dominated by moist convection. There, warming following the moist adiabat appears to be a reasonable approximation (Romps 2011). However, there is no consensus in literature on the change in thermal stratification for Northern Hemispheric midlatitudes (e.g., Frierson 2006; Schneider and O'Gorman 2008). The change in thermal stratification with warming is likely to vary with latitude.

Up to now, we have assumed warming to occur homogeneously with height, so that the temperature perturbation at each height equals the surface temperature perturbation, maintaining the dry stability of the profile. This is not the case for a moist adiabatic perturbation, which leads to stabilization of the profile as the moist adiabatic lapse rate decreases with warming. With this stabilization of the environmental profile, precipitation scaling is expected to be lower than that found in section 4 . In this section we analyze how the scaling of precipitation intensity is affected by changing stability and assess whether the choice of perturbing the climate by a constant amount is justified over the Netherlands.

To determine how precipitation intensity scaling changes when the environmental stability increases with warming, we have systematically perturbed the climate with increasing stability from the constant temperature perturbation $\left(c_{0}=1\right)$ to the moist adiabatic temperature perturbation $\left(c_{0}=0\right)$, following

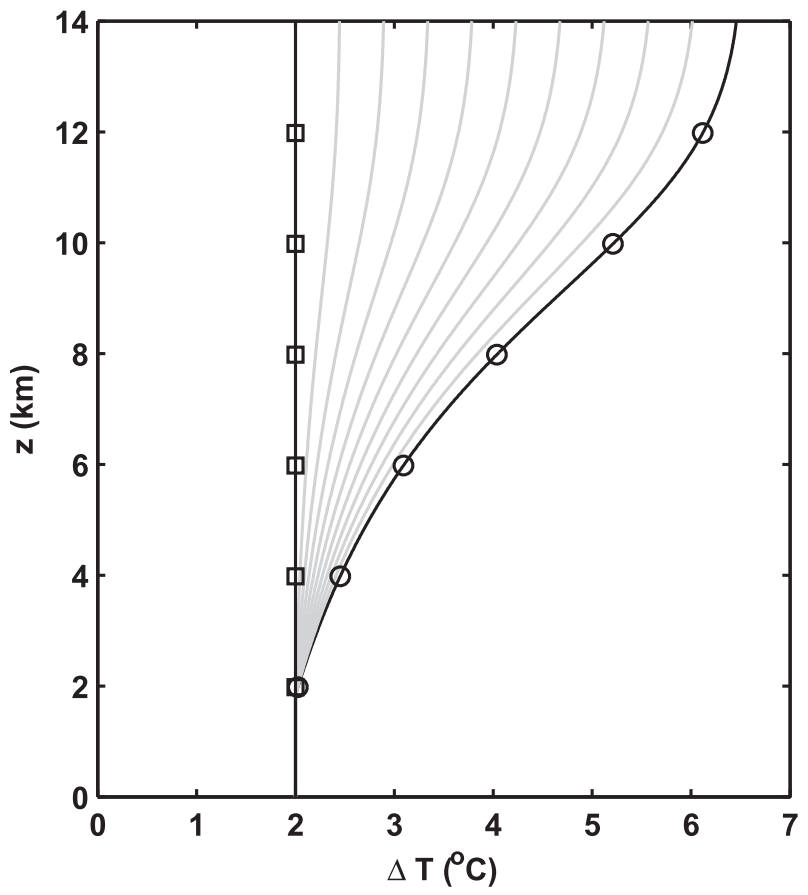

FIG. 9. Temperature perturbations from $c_{0}=0$ (moist adiabatic; black circles) to $c_{0}=1$ (constant with height; black squares). The perturbations based on a linear combination of these two perturbation types, that is, for $c_{0}=[0,1]$, are shown in gray.

$$
T_{e}^{\prime}=T_{e}+c_{0} \Delta T_{0}+\left(1-c_{0}\right) \Delta T_{m} .
$$

Here, $T_{e}^{\prime}$ indicates the perturbed environmental temperature profile, which is now described by linear combination of the constant temperature perturbation $\Delta T_{0}$ and the moist adiabatic temperature perturbation $\Delta T_{m}$, with $c_{0}$ being a constant varying from zero to one. For the moist adiabatic temperature perturbation, the environmental temperature is increased by $\Delta T_{m}$ starting at the LCL, where $\Delta T_{m}$ is the difference between the moist adiabatic profiles based on the original surface temperature $T_{0, e}$, and the perturbed surface temperature $T_{0, e}+$ $\Delta T_{0}$. Below the LCL, the temperature perturbation is kept constant at $\Delta T_{0}$. Figure 9 shows the $2^{\circ} \mathrm{C}$ temperature perturbations ranging from $\Delta T_{0}$ to $\Delta T_{m}$ with height, which ranges from $2^{\circ} \mathrm{C}$ at the surface, to $6.5^{\circ} \mathrm{C}$ at the top of the troposphere, clearly showing the stabilization effect of the moist adiabatic adjustment.

The change in stability from the moist adiabatic to constant perturbation with height is visualized in Fig. 10, which shows the phase space of CAPE in the domain of $c_{0}$ versus $\Delta T_{0}$, that is, the perturbation step versus perturbation type. Since the $c_{0}=0$ perturbation is based on the same adiabats as the parcel, CAPE is unaffected by the perturbation step in this case. As $c_{0}$ increases, the profile becomes less stable, and CAPE will increase with 


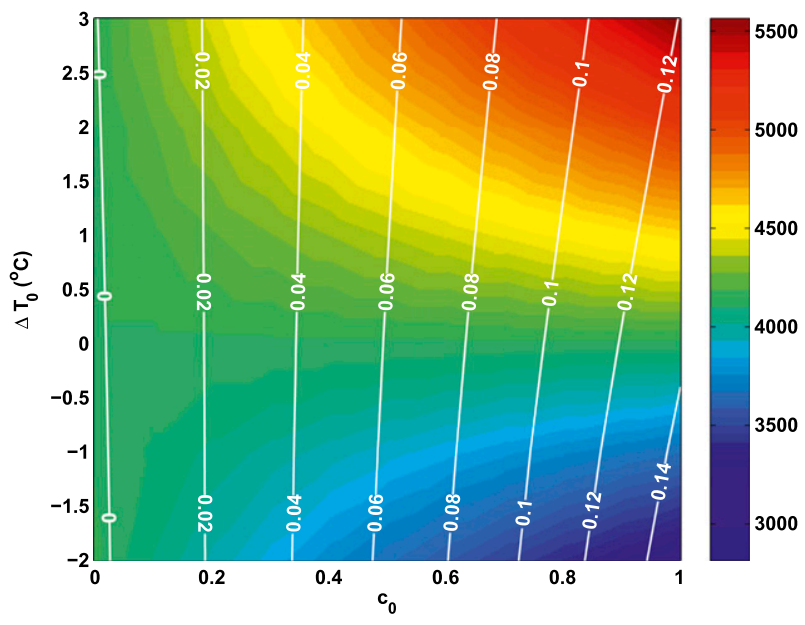

FIG. 10. The phase space of CAPE $\left(\mathrm{J} \mathrm{kg}^{-1}\right)$ as a function of surface perturbation and perturbation type. Here, $c_{0}=0$ represents a purely moist adiabatic perturbation, while $c_{0}=1$ represents a perturbation that is constant with height. The contours of the fractional rate of change of CAPE are overlain in white.

increasing $\Delta T_{0}$. This increase becomes more pronounced as the stratification destabilizes. The growth rate of CAPE is also visualized. It shows that the increase in CAPE is more or less unaffected by the perturbation step and increases from $0 \%{ }^{\circ} \mathrm{C}^{-1}$ for $c_{0}=0$ to approximately $14 \%{ }^{\circ} \mathrm{C}^{-1}$ for $c_{0}=1$.

Figure 11 shows the precipitation intensity increase in the phase space of $\Delta T_{0}$ versus $c_{0}$, where scaling is performed for the entraining plume. The precipitation scaling is fairly constant with perturbation step, which is in line with Fig. 6. The precipitation rate ranges from about $6 \%{ }^{\circ} \mathrm{C}^{-1}$ for the moist adiabatic adjustment to just under $10 \%{ }^{\circ} \mathrm{C}^{-1}$ for the constant temperature perturbation with height. As expected, scaling is lower when profile perturbations show increasing stabilization with height. Interestingly, the scaling for the moist adiabatic perturbation is close to CC scaling, which is in agreement with precipitation scaling in the tropics as found by Muller et al. (2011) and Romps (2011). The figure also contains contours of the ratio of precipitation scaling for diluted versus undiluted ascent. Though scaling is more or less unaffected by entrainment for the constant perturbation with height, the difference between diluted and undiluted precipitation scaling increases as perturbations come closer to the moist adiabatic perturbation. These results show that the increase of precipitation rate is strongly influenced by how the temperature is perturbed.

Next, we determine whether the constant temperature perturbation is a reasonable choice for extreme precipitation over the Netherlands. For comparison, we analyze the data of an eight-member downscaling with

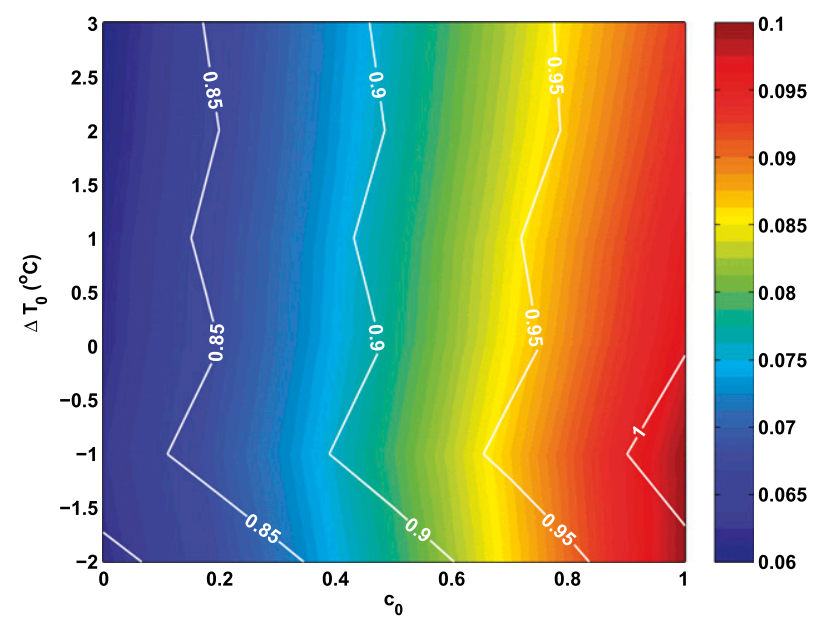

FIG. 11. The phase space of the precipitation rate scaling $\left({ }^{\circ} \mathrm{C}^{-1}\right)$ for diluted ascent as a function of surface perturbation and perturbation type. Here, $c_{0}=0$ represents a purely moist adiabatic perturbation, while $c_{0}=1$ represents a perturbation that is constant with height. The ratio of the increase in precipitation rate for diluted over undiluted ascent is depicted in white.

the Regional Atmospheric Climate Model, version 2RACMO2 (Van Meijgaard et al. 2008), at 12-km resolution on a domain over western Europe, which is driven by the global model EC-Earth (Hazeleger et al. 2012). The model configuration of the runs with the global model are identical to those performed for phase 5 of the Coupled Model Intercomparison Project (CMIP5) (Taylor et al. 2012) and use the Representative Concentration Pathway 8.5 (RCP8.5) greenhouse gas scenario. The period 2041-70 is compared to 1981-2010, during which the global-mean-temperature rise reaches $2^{\circ} \mathrm{C}$. Profile data are available at seven points in the Netherlands. Since we are interested in extremes, a further selection is made of the 1000 profiles with the highest CAPE for each summer month [JuneAugust (JJA)].

Figure 12 shows the temperature perturbation for a data selection based on the profiles with the highest CAPE in the summer months. The perturbation profile is fairly constant with height up to about $750 \mathrm{hPa}$, decreases by about $0.5^{\circ} \mathrm{C}$ to about $650 \mathrm{hPa}$, and then increases to close to its surface value at $300 \mathrm{hPa}$, after which it dies out. Though the perturbation is not constant with height, deflections from the surface perturbation are less than $1^{\circ} \mathrm{C}$, in both positive and negative directions. The use of a perturbation constant in height thus seems reasonable. Furthermore, the increase of CAPE found for (near-) constant temperature perturbations is consistent with the CAPE increase found for the output of the RACMO2 climate analysis for the Netherlands. Apparently, the change in stability is well 


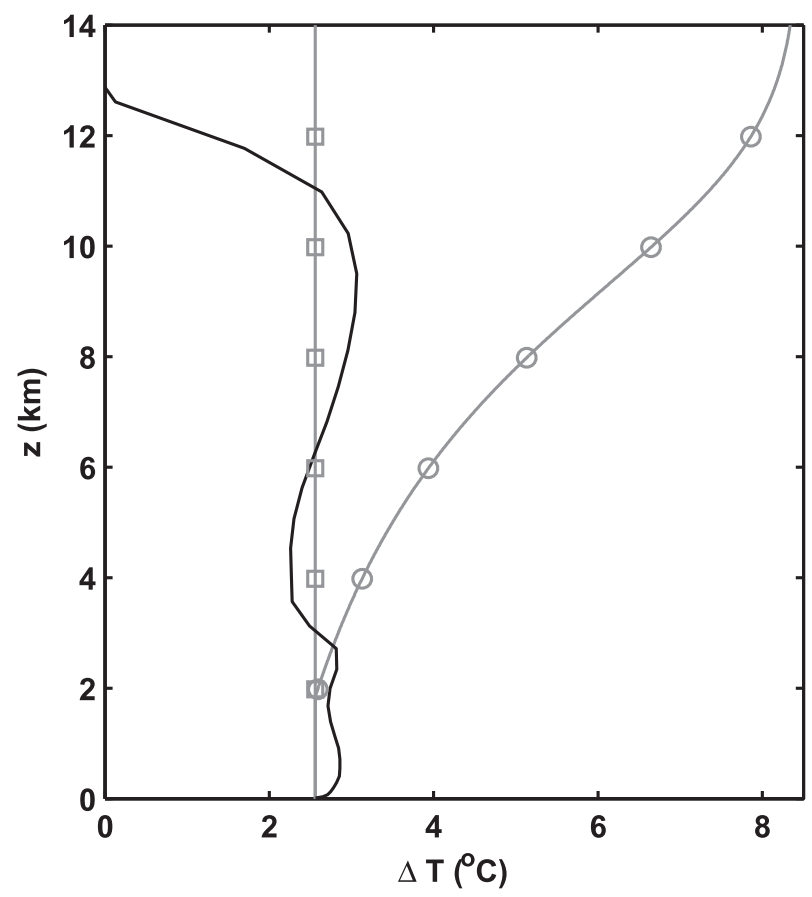

FIG. 12. RACMO2 temperature perturbation profile, based on the temperature increase of the 1000 profiles with the highest CAPE per month for June, July, and August. For reference, the constant (gray squares) and moist adiabatic (gray circles) perturbations corresponding to the surface perturbation temperature of the RACMO2 profile have been added.

portrayed by this type of perturbation over the Netherlands.

Overall, this analysis has provided important insights in stability changes and precipitation scaling. Comparison with climate run data indicates that a perturbation maintaining the tropospheric thermal stratification is justified over the Netherlands. Furthermore, with superCC scaling for the perturbation that is constant with height and CC scaling for the moist adiabatic perturbation, it is clear that the perturbation intensity increase is dependent on the change in environmental stability.

\section{Summary and discussion}

This paper offers a comprehensive analysis of extreme precipitation intensity increase over the Netherlands. First, a temporal analysis of precipitation data over the Netherlands is used to verify that the previously found 2CC dependency of hourly extreme precipitation on surface moisture is robust for convective events. To understand this dependency, a frequently used scaling based on the column condensation rate is analyzed in depth by means of an entraining plume model. Scaling analysis shows that the precipitation rate increase is determined by both the integrated lateral moisture flux and the cloud-base moisture flux. A decomposition into thermodynamic and dynamic effects further enhances our understanding of how this scaling is affected by different parameters. Finally, a sensitivity study of environmental stability perturbations indicates that precipitation scaling is influenced by how the stability changes with warming.

At an hourly resolution, previous studies have shown bifurcated scaling behavior for extreme precipitation over the Netherlands, with CC scaling for low surface moisture and temperature and 2CC scaling at higher values. Until now, it has remained unclear whether the observed 2CC dependency describes the scaling of convective extremes or if this behavior is a statistical artifact induced by the transition between the stratiform and convective regimes that both scale with CC. By analyzing extreme scaling with respect to surface moisture at three different temporal resolutions, we have attempted to resolve this. While hourly precipitation extreme scaling is analogous to previous work, CC scaling is found for the daily resolution, where stratiform precipitation dominates the extremes. By analyzing subhourly precipitation, convective events are selected from the dataset, leading to a $2 \mathrm{CC}$ trend over the entire range of dewpoint temperatures. This places hourly scaling relations into context, showing that $2 \mathrm{CC}$ is not a statistical artifact. We instead confirm that while CC scaling applies for stratiform extremes, 2CC scaling is a robust relation for convective extreme precipitation in the Netherlands.

Using a basic entraining plume model, we have attempted to reproduce the 2CC dependency based on the scaling of the condensation rate as proposed in previous studies. Though the plume model does not replicate $2 \mathrm{CC}$, it produces a robust, super-CC condensation rate increase just below $10 \%{ }^{\circ} \mathrm{C}^{-1}$. Changes in entrainment only have a minor effect on this. Two processes are responsible for the scaling that we find: the integrated lateral moisture convergence, with approximately $2 \mathrm{CC}$, and the cloud-base moisture flux, which shows an increase slightly greater than CC scaling. With a weight of $54 \%$ versus $46 \%$, respectively, the lateral moisture convergence and the cloud-base moisture flux contribute to the increase of precipitation rate almost equally. These relative contributions are controlled by the unperturbed vertical velocity and specific humidity profiles. Decomposition into thermodynamic (perturbed humidity) and dynamic (perturbed vertical velocity) contributions show that thermodynamic scaling is related to surface CC scaling through the cloud-base moisture flux and to WVP scaling through the integrated lateral moisture convergence, leading to a total thermodynamic contribution of about $7 \%{ }^{\circ} \mathrm{C}^{-1}$. An 
additional $3 \%{ }^{\circ} \mathrm{C}^{-1}$ is provided by the dynamic scaling contribution, based on changes in vertical velocity.

The scaling analysis of precipitation increase illustrates the important role of the vertical velocity. Muller et al. (2011) find a negative dynamic contribution. In their experiment, which uses radiative-convective equilibrium, vertical velocity increases in the upper part of the profile but decreases in the lower part, which has the most weight because of the shape of the humidity gradient. However, in this experiment the dynamic contribution is always positive, simply because of the way that $w$ has been defined. Apart from determining dynamic scaling, the vertical velocity also determines the importance of the integrated lateral moisture flux with respect to the cloud-base flux. Assuming the dynamic scaling to be unchanged, alterations in $w$ can theoretically lead to scaling results in the range of approximately $\mathrm{CC}$ up to $2 \mathrm{CC}$ scaling. To understand extreme precipitation scaling therefore requires a deeper understanding in both shape and evolution of the vertical velocity.

The influence of environmental stability change on extreme intensity scaling is assessed by a sensitivity analysis, where the environmental temperature is increased by a range of moist adiabatic to constant temperature perturbations. Use of the constant perturbation for the Netherlands is supported by comparison to RACMO2 climate data, while a moist adiabatic perturbation might be more valid for the tropics. Precipitation scaling varies with stability from CC scaling for the moist adiabatic perturbation to super-CC scaling for the constant temperature perturbation with height. The CC scaling is in line with the intensity increase found for CRM studies in the tropics (Romps 2011; Muller et al. 2011). These results thus imply that there could be a latitudinal effect on the scaling of extreme precipitation, which depends on how the thermal stratification changes with global warming. This is an important point which deserves more attention.

The entraining plume model is obviously a very simple representation of reality. This leads to shortcomings in the dynamical contributions to the rate of change of the precipitation intensity. For example, in nature the dynamics of the subcloud layer are coupled to the dynamics of the cloud, as downdrafts generated in the cloud influence the subcloud layer. This mechanism is not present in the model, since there is no transfer of information from high to lower levels, as is sometimes done by using a scheme such as CAPE closure. This may have consequences for the dynamical contribution at cloud base. Furthermore, unlike cloud-resolving models, the plume model has fixed environmental conditions. Processes that might affect these conditions are not taken into account. While the plume model has provided us with useful insights into the scaling behavior of extreme precipitation, we therefore think that to go further in this analysis, using a model that resolves $3 \mathrm{D}$ dynamics is needed.

Acknowledgments. The authors thank Erik van Meijgaard and Jisk Attema for providing us with the RACMO profile data. The authors are grateful to Steef Böing for useful discussions. This study was funded by Knowledge for Climate theme 6.

\section{REFERENCES}

Allen, M. R., and W. J. Ingram, 2002: Constraints on future changes in climate and the hydrological cycle. Nature, 419, 224-232.

Berg, P., and J. O. Haerter, 2011: Unexpected increase in precipitation intensity with temperature-A result of mixing of precipitation types? Atmos. Res., 119, 56-61.

— C. Moseley, and J. O. Haerter, 2013: Strong increase in convective precipitation in response to higher temperatures. Nat. Geosci., 6, 181-185.

de Roode, S. R., A. P. Siebesma, H. J. J. Jonker, and Y. de Voogd, 2012: Parameterization of the vertical velocity equation for shallow cumulus clouds. Mon. Wea. Rev., 140, 2424-2436.

de Rooy, W., and A. P. Siebesma, 2008: A simple parameterization for detrainment in shallow cumulus. Mon. Wea. Rev., 136, 560576.

Emori, S., and S. J. Brown, 2005: Dynamic and thermodynamic changes in mean and extreme precipitation under changed climate. Geophys. Res. Lett., 32, L17706, doi:10.1029/ 2005 GL023272.

Frierson, D. M. W., 2006: Robust increases in midlatitude static stability in simulations of global warming. Geophys. Res. Lett., 33, L24816, doi:10.1029/2006GL027504.

Haerter, J. O., and P. Berg, 2009: Unexpected rise in extreme precipitation caused by a shift in rain type? Nat. Geosci., 2, 372-373.

Hardwick-Jones, R., S. Westra, and A. Sharma, 2010: Observed relationships between extreme sub-daily precipitation, surface temperature, and relative humidity. Geophys. Res. Lett., 37, L22805, doi:10.1029/2010GL045081.

Hazeleger, W., and Coauthors, 2012: Ec-earth v2. 2: Description and validation of a new seamless earth system prediction model. Climate Dyn., 39, 2611-2629.

Holloway, C. E., and J. D. Neelin, 2009: Moisture vertical structure, column water vapor and tropical deep convection. J. Atmos. Sci., 66, 1665-1683.

Iribarne, J. V., and W. L. Godson, 1981: Atmospheric Thermodynamics. Kluwer, $259 \mathrm{pp}$.

Kessler, E., 1969: On Distribution and Continuity of Water Substance in Atmospheric Circulation. Meteor. Monogr., No. 10, Amer. Meteor. Soc., 84 pp.

Lenderink, G., and E. van Meijgaard, 2008: Increase in hourly precipitation extremes beyond expectations from temperature changes. Nat. Geosci., 1, 511-514.

$\longrightarrow$, and —, 2010: Linking increases in hourly precipitation extremes to atmospheric temperature and moisture changes. Environ. Res. Lett., 5, 025208, doi:10.1088/ 1748-9326/5/2/025208.

- H. Y. Mok, T. C. Lee, and G. J. van Oldenborgh, 2011: Scaling and trends of hourly precipitation extremes in two 
different climate zones-Hong kong and the Netherlands. Hydrol. Earth Syst. Sci., 15, 3033-3041.

Maeda, E. E., N. Utsumi, and T. Oki, 2012: Decreasing precipitation extremes at higher temperatures in tropical regions. Nat. Hazards, 64, 935-941.

Min, S.-K., X. Zhang, F. W. Zwiers, and G. C. Hegerl, 2011: Human contribution to more-intense precipitation extremes. Nature, 470, 378-381.

Muller, C. J., L. E. Back, and P. A. O'Gorman, 2011: Intensification of precipitation extremes with warming in a cloud-resolving model. J. Climate, 24, 2784-2800.

O'Gorman, P. A., 2012: Sensitivity of tropical precipitation extremes to climate change. Nat. Geosci., 5, 697-700.

_ , and T. Schneider, 2009a: The physical basis for increases in precipitation extremes in simulations of 21st-century climate change. Proc. Natl. Acad. Sci. USA, 106, 14773-14777.

O'Gorman, P., and T. Schneider, 2009b: Scaling of precipitation extremes over a wide range of climates simulated with an idealized GCM. J. Climate, 22, 5676-5685.

Pall, P., M. Allen, and D. Stone, 2007: Testing the ClausiusClapeyron constraint on changes in extreme precipitation under $\mathrm{CO}_{2}$ warming. Climate Dyn., 28, 351-363.

— , T. Aina, D. A. Stone, P. A. Stott, T. Nozawa, A. G. J. Hilberts, D. Lohmann, and M. R. Allen, 2011: Anthropogenic greenhouse gas contribution to flood risk in England and Wales in autumn 2000. Nature, 470, 382-385.

Romps, D. M., 2011: Response of tropical precipitation to global warming. J. Atmos. Sci., 68, 123-138.

Schneider, T., and P. A. O'Gorman, 2008: Moist convection and the thermal stratification of the extratropical troposphere. $J$. At mos. Sci., 65, 3571-3583.

Sherwood, S. C., W. Ingram, Y. Tsushima, M. Satoh, M. Roberts, P. L. Vidale, and P. A. O'Gorman, 2010: Relative humidity changes in a warmer climate. J. Geophys. Res., 115, D09104, doi:10.1029/2009JD012585.

Siebesma, A. P., 1998: Shallow cumulus convection. Buoyant Convection in Geophysical Flows, E. J. Plate, et al., Eds., Kluwer, 441-486.

— , and A. M. Holtslag, 1996: Model impacts of entrainment and detrainment rates in shallow cumulus convection. J. Atmos. Sci., 53, 2354-2364.

Singleton, A., and R. Toumi, 2012: Super-Clausius-Clapeyron scaling of rainfall in a model squall line. Quart. J. Roy. Meteor. Soc.

Sundqvist, H., 1978: A parameterization scheme for non-convective condensation including prediction of cloud water content. Quart. J. Roy. Meteor. Soc., 104, 677-690.

Taylor, K. E., R. J. Stouffer, and G. A. Meehl, 2012: An overview of CMIP5 and the experiment design. Bull. Amer. Meteor. Soc., 93, 485-498.

Tiedtke, M., 1989: A comprehensive mass flux scheme for cumulus parameterization in large-scale models. Mon. Wea. Rev., 117, 1779-1800.

Trenberth, K. E., A. Dai, R. Rasmussen, and D. Parsons, 2003: The changing character of precipitation. Bull. Amer. Meteor. Soc., 84, 1205-1217.

Utsumi, N., S. Seto, S. Kanae, E. E. Maeda, and T. Oki, 2011: Does higher surface temperature intensify extreme precipitation? Geophys. Res. Lett., 38, L16708, doi:10.1029/2011GL048426.

Van Meijgaard, E., L. H. Van Ulft, W. J. van de Berg, F. C. Bosveld, B. J. J. M. Van den Hurk, G. Lenderink, and A. P. Siebesma, 2008: The KNMI regional atmospheric climate model RACMO version 2.1. Koninklijk Nederlands Meteorologisch Instituut Rep. TR-302, 43 pp.

Weisman, M. L., and J. B. Klemp, 1982: The dependence of numerically simulated convective storms on vertical wind shear and buoyancy. Mon. Wea. Rev., 110, 504-520. 